\title{
Neuropathological abnormalities of the corpus callosum in schizophrenia: a diffusion tensor imaging study
}

\author{
J Foong, M Maier, C A Clark, G J Barker, D H Miller, M A Ron
}

\begin{abstract}
Objectives-Diffusion tensor imaging (DTI), a technique capable of examining water diffusion in different tissues and the organisation of white matter tracts, was used to investigate the neuropathology of the corpus callosum in vivo in patients with schizophrenia.

Methods-Diffusion tensor imaging was performed in 20 schizophrenic patients and 25 healthy controls. Two complementary measures, mean diffusivity and fractional anisotropy, which are considered to be sensitive indices of axonal integrity, were obtained from regions of interest in the genu (anterior) and splenium (posterior) of the corpus callosum.

Results-Mean diffusivity was significantly increased and fractional anisotropy significantly reduced in the splenium but not the genu of the corpus callosum in the schizophrenic group compared with controls. There were no significant sex differences in the DTI measures for either the schizophrenic or control group. Clinical variables such as age, duration of illness, dose of antipsychotic medication, and schizophrenic symptoms did not predict the DTI changes in the schizophrenic patients.
\end{abstract}

Conclusions-The presence of DTI changes in the splenium but not the genu of the corpus callosum suggests that there may be a focal disruption of commisural connectivity in schizophrenia. However, these findings do not exclude the possibility of abnormalities in other areas of the corpus callosum or other regions of white matter and further research using different methods of analysis may enable us to clarify this. Diffusion tensor imaging is a valuable tool in investigating the structure of white matter in schizophrenia.

(F Neurol Neurosurg Psychiatry 2000;68:242-244)

Keywords: corpus callosum; diffusion tensor imaging; schizophrenia

Structural abnormalities of the corpus callosum in schizophrenia have been documented and a meta-analysis ${ }^{1}$ has established that most MRI studies have found a reduction in the area of the corpus callosum in schizophrenic patients compared with controls. However, less is known about the pathology or the functional significance of these structural abnormalities. Earlier postmortem studies ${ }^{23}$ found no abnormality in axonal counts but a more recent study has reported disruption of axonal density in most areas of the corpus callosum in female schizophrenic patients. ${ }^{4}$

Diffusion tensor imaging (DTI), a noninvasive MRI technique, is capable of examining water diffusion and the organisation of fibres in white matter tracts. Water diffusion in tissue is modified by its structural environment; in white matter, water diffusion is greater along fibre tracts than across them resulting in anisotropic diffusion. ${ }^{56}$ This technique provides quantitative measures of directionally averaged diffusion (mean diffusivity) and diffusion anisotropy (fractional anisotropy). Increased mean diffusivity has been reported in multiple sclerosis lesions and normal appearing white matter which may reflect oedema, demyelination, and axonal loss. ${ }^{7}$ Reduced fractional anisotropy provides further evidence of structural damage and may reflect a disruption in the organisation of tracts. ${ }^{78}$ These two complementary measures are therefore considered to be sensitive indices of axonal integrity. The aim of our study was to investigate the neuropathology of the corpus callosum in schizophrenia using DTI.

\section{Methods}

SUBJECTS

Twenty patients ( 15 men, five women) with a mean age of 37.65 years and who fulfilled the DSM IV diagnosis of schizophrenia were recruited from the Maudsley and Bethlem Hospitals for the study. Their mean duration of psychiatric symptoms was 13.75 years and they had a history of exposure to neuroleptic medication. All patients were on antipsychotic medication at the time of the study (mean dosage $367.5 \mathrm{mg}$ per day equivalent to chlorpromazine, British National Formulary 1998). Psychiatric symptoms were assessed using the positive and negative syndrome scale (PANSS). ${ }^{9}$ 

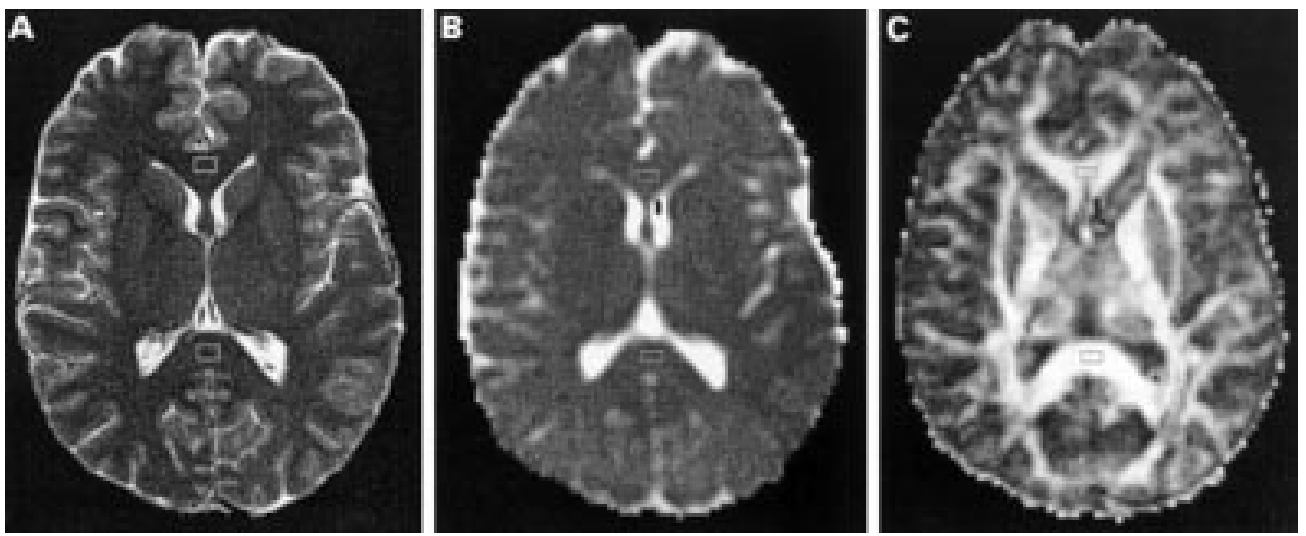

Regions of interest in the corpus callosum (genu and splendium); (A) echoplanar imaging; (B) mean diffusivity; (C) fractional anisotropy.

Twenty five controls (16 men, nine women) with a mean age of 33.84 years were selected to match the patient group for sex and age.

Any subjects with a history of neurological or systemic illness, head injury, and drug or alcohol misuse were excluded from the study. All subjects were assessed to be right handed apart from one patient and one control, using the Annett questionnaire ${ }^{10}$ for hand preference. Informed consent was obtained from all subjects.

MRI

Diffusion tensor imaging was performed on a Signa 1.5 Tesla scanner (GE, Milwaukee, USA) equipped with shielded magnetic field gradients of up to $22 \mathrm{mT} / \mathrm{m}$. A standard quadrature head coil was used for RF transmission and reception of the NMR signal. Head motion was minimised with standard foam pad immobilisation as provided by the manufacturer. Diffusion weighted echo planar images (DW$\mathrm{EPI})$ were acquired in the axial plane $(\mathrm{TE}=78$ ms, $96 \times 96$ matrix, field of view $=24 \mathrm{~cm} \times 24 \mathrm{~cm}$, slice thickness $=5 \mathrm{~mm}$ ) at 12 slice locations centred on the lateral ventricles. The diffusion sensitising gradients were applied along seven non-collinear directions at four gradient strengths corresponding to $\mathrm{b}$ values from 0 to $700 \mathrm{~s} / \mathrm{mm}^{2}$. Images were acquired in two sets of contiguous interleaved slices due to image storage limitations. Five acquisitions of each set were performed and co-added after magnitude reconstruction to improve signal to noise ratio. Cardiac gating was utilised via a peripheral pulse oximeter; image acquisition was triggered from every second $\mathrm{R}$ wave to reduce signal modulation due to brain pulsation. Maps of the diffusion tensor elements, mean diffusivity (D), and fractional anisotropy (FA) were calculated on a pixel by pixel basis as described by Basser et al. ${ }^{11}$

Mean (SD) diffusivity and fractional anisotropy

\begin{tabular}{llll}
\hline & Schizophrenic $(n=20)$ & Control $(n=25)$ & Mann-Whitney \\
\hline D (splenium) & $0.943(0.138)$ & $0.840(0.073)$ & $\mathrm{z}=-2.51, \mathrm{p}<0.01$ \\
D (genu) & $0.808(0.067)$ & $0.804(0.053)$ & NS \\
FA (splenium) & $0.776(0.047)$ & $0.820(0.067)$ & $\mathrm{z}=-2.36, \mathrm{p}<0.02$ \\
FA (genu) & $0.820(0.057)$ & $0.823(0.047)$ & NS \\
\hline
\end{tabular}

$\mathrm{D}=$ mean diffusivity $\left(\mathrm{x} 10^{3} \mathrm{~mm}^{2} / \mathrm{s}\right)$.

$\mathrm{FA}=$ fractional anisotropy (dimensionless units).
IMAGE ANALYSIS

Images were displayed on a Sun workstation and the regions of interest (ROIs), standardised at $28.1 \mathrm{~mm}^{2}$, were outlined on the non-diffusion weighted $(b=0)$ echoplanar images and not directly on the DTI images to avoid any bias in placing them. The ROIs were placed in the genu (anterior) and splenium (posterior) of the corpus callosum on the slice in which they were visualised to be of maximal thickness. Adjacent slices were checked to ensure that partial volume effects from CSF were minimised. As DTI images were coregistered with the echoplanar images, the ROIs were automatically transferred to the corresponding diffusion maps to obtain D and FA measurements (figure).

STATISTICAL ANALYSIS

Non-parametric tests (Mann-Whitney, Spearman's correlation coefficient) were used for group comparisons and the correlation analysis. Regression analysis was applied to determine if clinical variables predicted the DTI changes.

\section{Results}

D was significantly increased and FA significantly reduced in the splenium of the corpus callosum in the schizophrenic group compared with controls $(p<0.01$ and $p<0.02$ respectively). However, D and FA in the genu did not significantly differ between the two groups. These results are shown in the table. In addition, there were no significant sex differences in D and FA for either the schizophrenic or control group.

Using forward regression analysis, none of the clinical variables - namely, age, duration of illness, dose of antipsychotic medication, positive or negative schizophrenic symptomspredicted the DTI changes in the schizophrenic group.

\section{Discussion}

We have used DTI to demonstrate that neuropathological abnormalities in the corpus callosum are present in schizophrenia. In this study, DTI changes were detected in the splenium but not the genu of the corpus callosum suggesting that these changes may be focal. 
Our study confirms the findings in a preliminary study ${ }^{12}$ which reported a reduction in FA in the corpus callosum in a small group of schizophrenic patients compared with non-age matched controls. In our sample, we selected age matched controls to compare with the patients as it has been demonstrated that there are age related changes in FA. ${ }^{13}$ We found a reduction in FA and an increase in $\mathrm{D}$ in the splenium suggesting a disruption of axonal integrity likely to be related to the density or organisation of fibres. It is possible that DTI changes may also reflect myelin abnormalities. However, a recent study ${ }^{14}$ demonstrated similar FA values in partially myelinated or unmyelinated white matter structures in the infant brain compared with the fully myelinated adult brain indicating that the contribution of myelin abnormalities to changes in anisotropy may be less significant than that from axonal abnormalities.

We chose to examine the corpus callosum using the ROI methodology in this study as it is a large fibre tract that can be easily identified and is large enough for the ROI to be reliably placed with minimal intersubject variability in the directionality and spread of fibre tracts. In the two areas of the corpus callosum sampled in this study, abnormalities were only present in the splenium and this makes it less likely that these changes are related to medication effects. It has been demonstrated that the interhemispheric fibres from the inferotemporal and occipital lobes transverse the splenium whereas those from the frontal lobes transverse the genu. ${ }^{15}$ It is therefore possible that the DTI changes in the splenium may have resulted from a focal disruption in the cortical neurons or axons in these areas. It remains possible that DTI abnormalities may have been present in wider regions of the corpus callosum, but the absence of changes in the genu suggest that these abnormalities may be focal within the corpus callosum. Sex differences in axonal density has been demonstrated in some areas of the corpus callosum ${ }^{4}$; however, we were not able to detect such differences in our sample, which may be due to the small number of women.

It would also have been of interest to examine other areas of white matter in the brain given that our previous work using magnetisation transfer imaging ${ }^{16}$ detected abnormalities in the temporal white matter in schizophrenic patients suggesting axonal and/or myelin disruption. Furthermore, recent studies using DTI in schizophrenic patients have reported widespread reduction in anisotropy in white matter $^{17}$ or more specifically, in prefrontal white matter. ${ }^{18}$ However, we did not consider using the ROI methodology in this study to be appropriate in examining other areas of white matter given the variability in the directionality of fibre tracts in regional white matter. Therefore, our findings in this study certainly do not exclude the possibility of abnormalities in other regions of white matter.

Age and duration of illness failed to predict the DTI changes in schizophrenic patients suggesting that these abnormalities are unlikely to be progressive although this would need to be confirmed by longitudinal studies. Likewise, positive and negative symptoms did not predict the DTI changes suggesting that they may be common to the different clinical subtypes. Our findings are unlikely to be medication related given that the dose of antipsychotic medication did not predict the DTI changes in these patients. We also attempted to minimise the possibility of alcohol and drug misuse as confounding factors and used this as one of the exclusion criteria for the study.

The findings of this study suggest that there may be a focal disruption of commissural connectivity in schizophrenia. However, it remains to be determined, using other methods of analysis, whether similar abnormalities can be detected in other areas of the corpus callosum or regional white matter. This study also confirms that DTI is a valuable tool in examining the structure of white matter in vivo in schizophrenia.

This study was funded by the Welcome Trust. Professor MA Ron was partly funded by the SCARFE Trust. We are grateful to members of the NMR Unit, Institute of Neurology, for their assistance.

1 Woodruff PWR, McManus IC, David AS. Meta-analysis of corpus callosum size in schizophrenia. 7 Neurol Neurosurg Psychiatry 1995;58:457-61.

2 Nasrallah HA, McCalley-Whitters M, Bigelow LB, et al. A histological study of the corpus callosum in chronic schizophrenia. Psychiatry Res 1983;8:251-60.

3 Casanova MF, Zito M, Bigelow LB, et al. Axonal counts of the corpus callosum of schizophrenic patients. $\mathcal{F}$ Neuropsychiatry 1989;1:391-3.

4 Highley JR, Esiri MM, McDonald B, et al. the size and fibre composition of the corpus callosum with respect to gender and schizophrenia: a post mortem study. Brain 1999;122: 99-110.

5 Chenervert TL, Brunberg JA, Pipe JG, et al. Anisotropic diffusion in human white matter: demonstration with MR techniques in vivo. Radiology 1990;177:401-5.

6 Pierpaoli C, Jezzard P, Basser PJ, et al. Diffusion tensor MR imaging of the human brain. Radiology 1996;201:637-48.

7 Werring DJ, Clark CA, Barker GJ, et al. The structural properties of multiple sclerosis lesions demonstrated by diffusion tensor imaging. ISMRM 1998;119.

8 Wieshmann UC, Symms MR, Franconi F, et al. Reduced diffusion anisotropy in malformations of cortical development. ISMRM 1998;1245.

9 Kay SR, Fiszbein A, Opler LA. The positive and negative syndrome scale (PANNS) for schizophrenia. Schizophrenia Bulletin 1987;13:261-76.

10 Annett MA. A classification of hand preference by association analysis. Br f Psychology 1970;61:303-21.

11 Basser PJ, Mattielo J, Le Bihan D. Estimation of the effective self-diffusion tensor from the NMR spin echo. $\mathcal{f}$ Magn Reson B 1994;103:247-54.

12 Hedehus M, de Crespigny A, Menon V, et al. Mapping of white matter tracts in schizophrenics using diffusion tensor imaging. ISMRM 1998;1342.

13 Virta A, Barnett A, Pierpaoli C. Diffusion anisotropy changes in normal aging. A study of the descending projection pathways at the level of the cerebral peduncle. ISMRM 1999:1347.

14 Miranda MJ, Born P, Wiegell MR. White matter tract visualization in infants by diffusion tensor MRI. ISMRM 1998; 528.

15 Pandya DN, Seltzer B. Two hemispheres - one brain: functions of the corpus callosum . In: Lepore F, Ptito M, Jasper $\mathrm{HH}$, eds. Neurology and neurobiology. Vol 17. New York: Alan R Liss, 1986. (Proceedings of the 6th International symAlan R Liss, 1986. (Proceedings of the 6th International symposium of the Centre of de Recherche en Sciences Neur
of the University of Montreal, May 16-18, 1984).

16 Foong J, Maier M, Barker GJ, et al. Investigating anatomical connectivity in schizophrenia using magnetization transfer maging [abstracts of the VIIth International Congress on Schizophrenia Research]. Schizophr Res 1999;36:197.

17 Lim KO, Hedehus M, Moseley M, et al. Compromised white matter tract integrity in schizophrenia inferred from diffusion tensor imaging. Arch Gen Psychiatry 1999;56: 367-74.

18 Buchsbaum MS, Tang CY, Peled S, et al. MRI white matter diffusion anisotropy and PET metabolic rate in schizophrenia. Neuroreport 1998;9:425-30. 